\title{
Satisfação com a Vida e Atitudes em Relação à Velhice de Idosos Frequentadores de Centros de Convivência em Função do Nível de Atividade Física
}

\author{
Satisfaction with Life and Attitudes in Relation to the Old Age of Frequenters of \\ Coexistence Centers in Function of the Physical Activity Level
}

\author{
Satisfacción con la Vida y Actitudes en Relación a la Vejez de los Ancianos \\ Frecuentadores del Centro de Convivencia en Función del Nivel de Actividad Física
}

\author{
Daniel Vicentini de Oliveira ${ }^{1}$ \\ Centro Universitário de Maringá \\ Jonatã Felipe Queiroz de Souza \\ Centro Universitário Metropolitano de Maringá \\ Carla Thamires Laranjeira Granja \\ Universidade Federal do Vale do São Francisco \\ Mateus Dias Antunes \\ Universidade de São Paulo \\ José Roberto Andrade do Nascimento Júnior \\ Universidade Federal do Vale do São Francisco
}

\begin{abstract}
Resumo
Estudo transversal, que teve como objetivo comparar a satisfação com a vida e as atitudes em relação à velhice de idosos frequentadores de centros de convivência em função do nível de atividade física (AF). Participaram 133 idosos, de ambos os sexos. Foi utilizado questionário sociodemográfico, o Mini Exame do Estado Mental, a Escala de Satisfação com a Vida, a Escala para Avaliação das Atitudes em Relação à Velhice e o Questionário Internacional de Atividade Física (International Physical Activity Questionnaire [IPAQ]), em sua versão curta. Os dados foram analisados por meio dos testes Kolmogorov-Smirnov e "U" de Mann-Whitney. Considerou-se um nível de significância de $p<0,05$. Os idosos ativos fisicamente são mais satisfeitos com a vida $(p=0,013)$ e possuem mais sentimentos de apreensão quanto ao futuro em relação à velhice $(p=0,002)$ do que os idosos irregularmente ativos. Conclui-se que, para os idosos frequentadores do centro de convivência do município de Sarandi, Paraná, PR, a prática de AF é um fator interveniente para a maior satisfação com a vida.
\end{abstract}

Palavras-chave: atividade motora, envelhecimento, satisfação pessoal

\begin{abstract}
This cross-sectional study aimed at comparing satisfaction with life and attitudes regarding old age of elderly people frecuenters of the coexistence centers according to the level of physical activity (PA). A total of 133 elderly people of both genders participated. A sociodemographic questionnaire was used, the Mini Mental State Examination, the Life Satisfaction Scale, the Scale for Assessing Attitudes Towards Old Age and the International Physical Activity Questionnaire (IPAQ), in its short version. Data were analyzed using the Kolmogorov-Smirnov test and the Mann-Whitney " $U$ " test. A level of significance of $p<0.05$ was considered. Physically active seniors are more satisfied with life $(p=$ 0.013 ) and have more feelings of apprehension about the future in relation to old age $(p=0.002)$ than irregularly active elderly. It is concluded that for elderly people frecuenters of the coexistence center in the city of Sarandi, Paraná, PR, the practice of PA is an intervening factor for greater satisfaction with life.
\end{abstract}

Keywords: motor activity, aging, personal satisfaction

\footnotetext{
${ }^{1}$ Endereço de contato: Avenida Guedner, 1610. CEP: 87050-900, Maringá, Paraná, Brasil. E-mail: d.vicentini@hotmail.com
} 


\section{Resumen}

Estudio transversal, que tuvo como objetivo comparar la satisfacción con la vida y las actitudes en relación a la vejez de ancianos frecuentadores de centros de convivencia en función del nivel de actividad física. Participaron 133 ancianos, de ambos sexos. Se utilizó cuestionario sociodemográfico, lo Mini Examen del Estado Mental, la Escala de Satisfacción con la Vida, la Escala para Evaluación de las Actitudes en Relación a la Vejez y el Cuestionario Internacional de Actividad Física (International Physical Activity Questionnaire [IPAQ]), en su versión corta. Los datos fueron analizados a través de las pruebas Kolmogorov-Smirnov y "U" de Mann-Whitney. Se consideró un nivel de significancia de $p<0,05$. Los ancianos activos físicamente son más satisfechos con la vida $(p=0,013)$ y poseen más sentimientos de aprehensión en cuanto al futuro en relación a la vejez $(p=0,002)$ que los ancianos irregularmente activos. Se concluye que, para los ancianos frecuentadores del centro de convivencia del municipio de Sarandi, Paraná, PR, la práctica de AF es un factor interviniente para la mayor satisfacción con la vida.

Palabras clave: actividad motora, envejecimiento, satisfacción personal

\section{Introdução}

A longevidade da população mundial é um acontecimento em crescente desenvolvimento, podendo ser explicado por diversos fatores, especialmente pelo avanço no conhecimento das ciências da saúde e pela dedicação dos profissionais responsáveis pela aplicação prática do conhecimento científico. Uma importante função da gerontologia é promover um envelhecimento saudável nos aspectos biológicos, psicológicos e sociais, visto que alcançar a satisfação com a vida na velhice não diz respeito somente a um desses fatores, mas sim a combinação dos três (Formiga et al., 2017).

A satisfação com a vida (SV) é um estado subjetivo que envolve a avaliação individual da própria vida como um todo, incluindo aspectos relacionados à saúde, família, amigos, trabaIho, moradia, relações sociais, entre outros (Sposito, D’Elboux, Neri, \& Guariento, 2013). A avaliação da satisfação está sujeita a uma comparação entre as condições de vida do indivíduo e um modelo por ele estabelecido (Silva, Amorim, Carvalho, \& Mesquita, 2017). Dessa forma, a SV pode ser considerada um indicador para políticas de saúde na velhice (Sposito et al., 2013).

Uma importante política pública voltada ao bem-estar e à satisfação com a vida da população idosa é a promoção de atividade física (AF). A AF proporciona melhores níveis de capacidade funcional (Rocha et al., 2017; Scarabottolo et al., 2017), força (Arruda, Bazaglia, Saravalli, Cassettari, \& Souza, 2014), equilíbrio corporal (Silva et al., 2017), além da diminuição dos índices de depressão (Teixeira, Nunes, Ribeiro, Arbinaga, \& Vasconcelos-Raposo, 2016; Mendes, Carvalho, Silva, \& Almeida, 2017), estresse (Freitas, Melo, Leopoldino, Boletini, \& Noce, 2017), ansiedade (Guimarães, Pedrini, Matte, Monte, \& Parcias, 2017), por exemplo.

Além disso, quando praticada em grupos (parques, praças, centros de convivência, entre outros ambientes), a AF pode proporcionar oportunidades de comunicação, confidência, além de sentimentos de segurança e apoio (Melo et al., 2017). As interações sociais são importantes para a quebra de certos estereótipos quanto à pessoa idosa, visto que o contato com pessoas de diferentes faixas etárias promove a desconstrução de preconceitos relacionados à idade (Tarallo, Neri, \& Cachioni, 2017). Desse modo, o idoso, ao ser enxergado sem estereótipos, faz uma auto percepção positiva que contribui em diversos aspectos, tornando suas atitudes em relação à velhice cada vez mais positivas.

Tais atitudes relacionadas à velhice se aprendem ao longo da vida em diferentes contextos sociais, a partir da convivência com idosos ou com o nosso próprio envelhecimento e, 
além disso, fazem parte de um campo conceitual que inclui as crenças, os preconceitos e os estereótipos (Neri, 2001). As atitudes são socialmente aprendidas e servem para prevenção, explicação, regulação e orientação dos pensamentos, sentimentos e ações particulares e gerais diante de pessoas, grupos e circunstâncias sociais (Tarallo et al., 2016; Krug, Lopes, Balbé, Marchesan, \& Mazo, 2016; Albano, Domingues, Abrantes, Corrêa, \& Camargos, 2017).

Diversos estudos já investigaram as atitudes em relação à velhice (Tarallo et al., 2016; Silva et al., 2017), a satisfação de vida de idosos (Sposito et al., 2013; Tomomitsu, Perracini, \& Neri, 2014) e o nível de atividade física de idosos (Benedetti, Mazo, \& Borges, 2012; Robins, Hill, Finch, Clemson, \& Haines, 2018), no entanto, não foram encontrados estudos que explorem essas três variáveis juntamente, sendo esta a lacuna que o presente estudo visa explorar. Desse modo, o presente estudo torna-se relevante, ao passo que a compreensão do envelhecimento e do bem-estar da população idosa poderá agregar conhecimentos importantes para o desenvolvimento do país (Formiga et al., 2017).

Diante desse cenário, o objetivo do presente estudo foi comparar a satisfação com a vida e as atitudes em relação à velhice de idosos frequentadores de centros de convivência em função do nível de atividade física.

\section{Métodos}

\section{Participantes}

Foram pesquisados 133 idosos (60 anos ou mais), de ambos os sexos, com capacidade de fala e audição preservadas, que permitiram a aplicação dos questionários e são frequentadores de centros de convivência do município de Sarandi, Paraná.

O Mini Exame do Estado Mental (MEEM) foi utilizado para excluir idosos com possíveis deficits cognitivos. O MEEM é composto por questões agrupadas em sete categorias: orientação temporal, orientação espacial, registro de três palavras, atenção e cálculo, recordação das três palavras, linguagem e capacidade visoconstrutiva (Folstein, Folstein, \& McHugh, 1975). As notas de corte utilizadas para exclusão pelo MEEM foram: 17 para os analfabetos; 22 para idosos com escolaridade entre 1 e 4 anos; 24 para os com escolaridade entre 5 e 8 anos e 26 para aqueles com 9 anos ou mais anos de escolaridade. Estes pontos de corte foram baseados nos critérios de Brucki, Nitrini, Caramelli, Bertolucci, e Okamoto (2003). Correspondem à média obtida por esses autores para cada faixa de escolaridade, menos um desvio padrão. Idosos classificados abaixo do ponto de corte específico para sua escolaridade foram excluídos.

\section{Instrumentos}

O perfil sociodemográfico e de saúde dos idosos foi avaliado por meio de um questionário semiestruturado elaborado pelos próprios autores, composto por questões referentes à faixa etária (60 a 69 anos; 70 anos ou mais); sexo (masculino; feminino); estado conjugal (com companheiro; sem companheiro); cor (branca; preta; amarela); situação ocupacional (ativa; inativa); renda mensal em salário mínimo (SM) de referência no Censo Demográfico 2017 do Instituto Brasileiro de Geografia e Estatística (IBGE) (menos de 1SM; 1 a 2 SM; mais de 2 SM); aposentadoria (sim ou não); escolaridade (analfabeto; Fundamental incompleto; 
Fundamental completo, Médio incompleto); percepção de saúde (boa, regular, ruim); quedas nos últimos seis meses (sim; não); tempo que frequenta o centro (menos de 1 ano; 1 a 2 anos; 3 anos ou mais); quantas atividades pratica no centro $(1 ; 2 ; 3)$; realização de exercício fora do centro (sim; não).

Para pesquisar a satisfação com a vida, foi utilizada a Escala de Satisfação com a Vida proposta por Diener, Emmons, Larsen, e Griffin (1985) e adaptada para o contexto brasileiro por Neto (1999). Esta se compõe de cinco itens, sendo as respostas graduadas de acordo com uma escala tipo Likert, que vai de 1 = discordo totalmente a 7 = concordo totalmente. Esta escala tem o propósito de avaliar o julgamento que as pessoas fazem acerca da sua própria satisfação com a vida, sendo elas próprias a eleger, de acordo com seus valores e interesses, os aspectos a serem considerados para expressar tal satisfação. De um possível escore total de 35 pontos, quanto mais próximo deste número, melhor a satisfação com a vida do idoso (Oliveira et al., 2009).

Em relação às atitudes em relação à velhice, foi utilizada a Escala para Avaliação de Atitudes em Relação à Velhice, originalmente elaborada por Sheppard (1981) e validada para a Língua Portuguesa por Neri (2001). O instrumento é composto por 20 itens que avaliam sentimentos de satisfação à velhice (autoestima, sexualidade, autorrealização, lazer e companheirismo nas questões 1 , 3, 4, 5, 6, 9, 12, 13, 15, 18, 19 e 20) e sentimento de perda em relação à velhice (incerteza, morte, dependência, solidão e apreensão quanto ao futuro nas questões $2,7,8,10,11,14,16,17)$. Os itens são respondidos em uma escala do tipo Likert de 5 pontos, de "discordo totalmente" (1) até "concordo totalmente" (5). Quanto maior a média de cada dimensão, maior é o grau de sentimento de satisfação à velhice ou de sentimento de perda em relação à velhice.

O nível de atividade física dos idosos foi avaliado por meio da versão curta do Questionário Internacional de Atividade Física (IPAQ) (Matsudo et al., 2001). Este instrumento avalia atividades físicas realizadas no tempo de lazer, como deslocamento de um lugar ao outro, serviços domésticos e atividades ocupacionais. Foi considerado o tempo de 150 minutos semanais de atividades físicas para o sujeito ser classificado como fisicamente ativo; para menos de dez minutos semanais, o sujeito foi considerado sedentário, e aqueles que realizam ao menos dez minutos, mas não alcançam os 150 minutos, foram consideradas insuficientemente ativos (Matsudo et al., 2001).

\section{Coleta de Dados}

Estudo observacional e transversal, aprovado pelo Comitê de Ética em Pesquisa com Seres Humanos da Faculdade Metropolitana de Maringá (FAMMA), por meio do parecer número 2.360.493/2017.

Os idosos voluntários foram abordados nos centros de convivência, pelos pesquisadores responsáveis, informados quanto à justificativa, objetivos e procedimentos a serem realizados, conforme orientações para pesquisa com seres humanos constantes na Resolução 196/96 do Conselho Nacional de Saúde. Após esses procedimentos, aqueles que aceitaram participar da pesquisa assinaram o Termo de Consentimento Livre e Esclarecido (TCLE).

Optou-se pela entrevista direta na aplicação dos questionários, em razão da possível dificuldade de leitura, problemas visuais e de compreensão dos questionamentos. 


\section{Análise de Dados}

Para a análise dos dados, inicialmente foi verificada a normalidade destes por meio do teste de Kolmogorov-Smirnov. Como os dados não apresentaram distribuição normal, foram utilizadas Mediana (Md) e Quartis (Q1; Q3) para a caracterização dos resultados. As variáveis categóricas foram descritas através da frequência absoluta ( $f$ ) e relativa (\%). Na comparação das variáveis em função do nível de atividade física, foi utilizado o teste " $U$ " de MannWhitney. Considerou-se um nível de significância de $p<0,05$.

\section{Resultados}

Nota-se (Tabela 1) a prevalência de idosos do sexo feminino (91,0\%), que não possuem companheiro (51,9\%), com mais de 70 anos $(52,6 \%)$, renda mensal de um a dois SM $(86,4 \%)$, da cor branca $(60,9 \%)$, aposentados $(82,0 \%)$ e com situação ocupacional inativa $(90,2 \%)$. Observou-se também que a maioria dos idosos possui Ensino Fundamental incompleto $(48,9 \%)$ e não estuda atualmente $(97,0 \%)$.

\section{Tabela 1}

Perfil Sociodemográfico dos Idosos Usuários dos Centros de Convivência de Sarandi, PR

\begin{tabular}{|c|c|c|}
\hline VARIÁVEIS & $F$ & $\%$ \\
\hline \multicolumn{3}{|l|}{ Sexo } \\
\hline Masculino & 12 & 9,0 \\
\hline Feminino & 121 & 91,0 \\
\hline \multicolumn{3}{|l|}{ Estado conjugal } \\
\hline Com companheiro & 64 & 48,1 \\
\hline Sem companheiro & 69 & 51,9 \\
\hline \multicolumn{3}{|l|}{ Faixa Etária } \\
\hline 60 a 69 anos & 63 & 47,4 \\
\hline 70 anos ou mais & 70 & 52,6 \\
\hline \multicolumn{3}{|l|}{ Renda mensal } \\
\hline Menos de $1 \mathrm{SM}$ & 15 & 12,0 \\
\hline 1 a 2 SM & 108 & 86,4 \\
\hline Mais de $2 \mathrm{SM}$ & 2 & 1,6 \\
\hline \multicolumn{3}{|l|}{ Escolaridade } \\
\hline Analfabeto & 36 & 27,1 \\
\hline Fundamental incompleto & 65 & 48,9 \\
\hline Fundamental completo & 22 & 16,5 \\
\hline Médio completo & 10 & 7,5 \\
\hline \multicolumn{3}{|l|}{ Cor } \\
\hline Branca & 81 & 60,9 \\
\hline Preta & 21 & 15,8 \\
\hline Amarela & 31 & 23,3 \\
\hline \multicolumn{3}{|l|}{ Aposentadoria } \\
\hline Não & 24 & 18,0 \\
\hline Sim & 109 & 82,0 \\
\hline \multicolumn{3}{|l|}{ Situação ocupacional } \\
\hline Ativa & 13 & 9,8 \\
\hline Inativa & 120 & 90,2 \\
\hline \multicolumn{3}{|c|}{ SM: salário mínimo de referência do ano de 2017 (R\$ 937,00). } \\
\hline
\end{tabular}


Já em relação ao perfil de saúde dos idosos usuários dos centros de convivência de Sarandi, PR (Tabela 2), verificou-se que a maioria dos idosos possui percepção de saúde regular (49,6\%), com pelo menos três doenças $(44,4 \%)$, não teve histórico de quedas $(75,9 \%)$, frequenta o centro de convivência há mais de três anos $(78,9 \%)$ e pratica duas atividades no centro de convivência $(47,4 \%)$. Nota-se que a maioria dos idosos apresentava nível de atividade física muito ativo / ativo (76,7\%).

\section{Tabela 2}

Perfil de Saúde e Atividade Física dos Idosos Usuários dos Centros de Convivência de Sarandi, $P R$

\begin{tabular}{lcc}
\hline VARIÁVEIS & $\boldsymbol{F}$ & $\%$ \\
\hline Percepção de saúde & 58 & 43,6 \\
$\quad$ Boa & 66 & 49,6 \\
$\quad$ Regular & 9 & 6,8 \\
$\quad$ Ruim & 34 & 25,6 \\
Quantidade de doenças & 40 & 30,1 \\
$\quad 1$ & 59 & 44,4 \\
2 & & \\
3 & 32 & 24,1 \\
Quedas nos últimos seis meses & 101 & 75,9 \\
Sim & & \\
$\quad$ Não & 12 & 9,0 \\
Tempo que frequenta o centro & 16 & 12,0 \\
$\quad$ Menos de 1 ano & 105 & 78,9 \\
1 a 2 anos & & \\
3 anos ou mais & 54 & 40,6 \\
Quantas atividades pratica & 63 & 47,4 \\
1 & 16 & 12,0 \\
2 & & \\
3 & 66 & 49,6 \\
Realiza exercício fora do centro & 67 & 50,4 \\
Sim & & 76,7 \\
Não & 102 & 23,3 \\
Nível de atividade física & 31 & \\
Muito ativo / ativo & & \\
Irregularmente ativo & & \\
\hline
\end{tabular}

Conforme os achados da Tabela 3, os idosos apresentaram as seguintes medianas nas variáveis psicológicas: satisfação com a vida $\mathrm{Md}=29,0$; expectativas quanto à atividade $\mathrm{Md}$ = 40,0; sentimentos em relação à velhice $M d=15,0$; expectativa quanto à satisfação $\mathrm{Md}=$ 12,0; e ansiedade em relação à morte $\mathrm{Md}=6,0$. 
Tabela 3

Percepção sobre a Satisfação com a Vida, Atitudes em Relação à Velhice e Prática de Atividade Física dos Idosos Usuários dos Centros de Convivência de Sarandi, PR

\begin{tabular}{lr}
\hline VARIÁVEIS & Md (Q1-Q3) \\
\hline Satisfação com a vida & $29,0(27,0-30,0)$ \\
Atitudes em relação à velhice & \\
Expectativas quanto à atividade & $40,0(39,0-41,0)$ \\
Sentimentos em relação à velhice & $15,0(12,0-16,0)$ \\
Expectativa quanto à satisfação & $12,0(11,0-12,5)$ \\
Ansiedade em relação à morte & $6,0(5,0-7,0)$ \\
Atividade física & \\
Dias de caminhada & $3,0(2,5-4,0)$ \\
Min. de caminhada p/ dia & $30,0(25,0-50,0)$ \\
Min. de caminhada p/ semana & $120(72,5-180,0)$ \\
Dias de atividade moderada & $3,0(2,0-3,0)$ \\
Min. de atividade moderada p/ dia & $20,0(15,0-25,0)$ \\
Min. de atividade moderada p/ semana & $60,0(40,0-77,5)$ \\
Dias de atividade vigorosa & $2,0(2,0-2,0)$ \\
Min. de atividade vigorosa p/ dia & $10,0(10,0-15,0)$ \\
Min. de atividade vigorosa p/ semana & $30,0(20,0-40,0)$ \\
\hline
\end{tabular}

Min.: minutos.

Em relação à prática de atividade física de intensidade vigorosa (Tabela 3), verificou-se mediana de 2,0 nos dias por semana, e 10,0 e 30,0 minutos, respectivamente, nos minutos por dia e semana. Nas atividades moderadas, verificou-se mediana de 3,0 nos dias por semana, e 20,0 e 60,0 minutos, respectivamente, nos minutos por dia e semana. Em relação à caminhada, os idosos apresentaram nos dias de caminhada mediana de 3,0, com mediana de 30,0 e 120,0 nos minutos de caminhada por dia e semana, respectivamente.

A Tabela 4 apresenta a comparação da satisfação com a vida e as atitudes em relação à velhice dos idosos em função do nível de atividade física.

Tabela 4

Comparação da Satisfação com a Vida e das Atitudes em Relação à Velhice dos Idosos em Função do Nível de Atividade Física

\begin{tabular}{|c|c|c|c|}
\hline \multirow{2}{*}{ VARIÁVEIS } & Muito ativo / ativo & Irregularmente ativo & \multirow{2}{*}{$\mathbf{P}$} \\
\hline & Md (Q1;Q3) & Md (Q1;Q3) & \\
\hline Satisfação com a vida & $29,0(27,0 ; 31,0)$ & $28,0(24,0 ; 29,0)$ & $0,002^{*}$ \\
\hline \multicolumn{4}{|l|}{ Atitudes em relação à velhice } \\
\hline Expectativas quanto à atividade & $40,0(39,0 ; 41,0)$ & $41,0(40,0 ; 41,0)$ & 0,471 \\
\hline Sentimentos em relação à velhice & $15,0(13,0 ; 16,0)$ & $13,0(11,0 ; 16,0)$ & $0,013^{*}$ \\
\hline Expectativa quanto à satisfação & $12,0(11,0 ; 13,0)$ & $12,0(11,0 ; 12,0)$ & 0,760 \\
\hline Ansiedade em relação à morte & $7,0(5,8 ; 7,0)$ & $6,0(5,0 ; 7,0)$ & 0,058 \\
\hline
\end{tabular}

* Diferença significativa: $p<0,05$ - Teste " $U$ " de Mann-Whitney. 
Houve diferença significativa (Tabela 4) entre os grupos apenas nos sentimentos em relação à velhice $(p=0,013)$ e na satisfação com a vida $(p=0,002)$. Tais achados indicam que os idosos ativos fisicamente são mais satisfeitos com a vida e possuem mais sentimentos de apreensão quanto ao futuro em relação à velhice do que os idosos irregularmente ativos.

\section{Discussão}

O presente estudo teve como objetivo comparar a satisfação com a vida e as atitudes em relação à velhice de idosos frequentadores de centros de convivência em função do nível de atividade física. Embora este seja um assunto em crescente atenção, ainda são necessárias evidências na literatura referentes aos papéis que a atividade física pode exercer sobre os atributos psicológicos na população idosa. Nessa perspectiva, os achados desta pesquisa contribuem para um conjunto de evidências, ao confirmar a presença de maior satisfação para os idosos que praticam atividade física.

Os achados demonstraram uma maior satisfação com a vida para os ativos fisicamente muito ativos ou ativos em relação aos irregularmente ativos. Tais resultados podem ser explicados devido aos inúmeros benefícios causados pela prática de atividade física, que corroboram para uma melhor avaliação individual da própria vida como um todo. Ainda, pelo fato de os participantes do presente estudo praticarem AF em grupo, não só os aspectos da satisfação com a vida relacionada à saúde sofrerão melhorias, como também os relacionados a amigos e relações sociais (Sposito et al., 2013).

Os sentimentos em relação à velhice também foram maiores para os idosos fisicamente ativos em relação aos irregulares. Esse resultado pode ser explicado devido à conotação de apreensão desses sentimentos, como os itens "Pensar na debilidade física que ocorre na velhice me aterroriza" e "Acho que vou sentir-me solitário na velhice", por exemplo. Dessa forma, o sentimento de que a velhice prenuncia dependência, solidão e morte podem servir como um fator motivacional para a prática de AF, visto que propiciará benefícios para sua independência nas atividades diárias, convívio social e longevidade (Formiga et al., 2017).

Em relação ao nível geral de satisfação com a vida, os idosos apresentaram uma ótima percepção de satisfação. Tais resultados revelam que os idosos possuem modos e motivos de viverem a vida de maneira positiva. Joia, Ruiz, e Donalisio (2007) argumentam que a saúde e independência são os principais determinantes na satisfação com a vida. Assim, visto que a maioria dos participantes do presente estudo é fisicamente ativa, esse resultado pode ser explicado pela prevenção e pelo tratamento de doenças, pelo aumento da capacidade funcional e por outros diversos benefícios suscitados pela prática regular de AF.

Nos aspectos voltados às expectativas quanto à atividade, os idosos demonstraram altos índices, evidenciando sua percepção de que é possível ser feliz, ativo e desfrutar a velhice. Em relação aos sentimentos de apreensão quanto ao futuro, expectativas em relação à satisfação e ansiedade em relação à morte, foram encontrados baixos escores. Tais resultados revelam que os idosos pouco percebem que a velhice prenuncia dependência, morte e solidão, tampouco consideram morrer cedo em vez de sentir a angústia e a solidão da velhice (Sposito et al., 2013). 
Ainda, a maioria dos idosos apresentou nível de atividade física ativo / muito ativo. Tal resultado foi esperado, visto que os participantes do estudo frequentavam um ambiente de promoção de AF. E, além disso, os idosos relataram realizar exercício fora desses centros. Em relação ao perfil de saúde e sociodemográfico, os achados do presente estudos corroboram com algumas investigações (Dawalibi, Goulart, \& Prearo, 2014; Krug et al., 2016).

\section{Conclusão}

Concluiu-se que, para os idosos frequentadores de centros de convivência do município de Sarandi, PR, a prática de AF é um fator interveniente para a maior satisfação com a vida e tal prática pode ser utilizada como motivação para idosos com grandes sentimentos de apreensão em relação à velhice. Ainda, os idosos apresentaram altos índices de satisfação com a vida e percepção da capacidade de ser feliz na velhice, bem como baixos sentimentos negativos quanto a ser idoso.

Por fim, os presentes resultados trazem dados relevantes para o entendimento das variáveis estudadas, porém, algumas limitações devem ser consideradas. Primeiramente, apenas uma cidade foi investigada e não possibilita a generalização dos resultados para todo o contexto brasileiro. Outra limitação importante se refere ao caráter transversal desta pesquisa, o que não permite fazer inferências de causalidade. Talvez um estudo longitudinal fosse capaz de apontar a natureza causal das associações entre as variáveis.

Dessa forma, para futuras investigações, sugerem-se principalmente estudos que investiguem tais associações por meio de um desenho de pesquisa longitudinal. Além disso, sugere-se a avaliação de outras cidades, outros tipos de atividade física e o uso de análises multiníveis, de modo que possa se entender as complexas relações entre essas variáveis em diferentes grupos.

\section{Referências}

Albano, D. C., Domingues, S. F., Abrantes, R., Corrêa, A. A. M., \& Camargos, G. L. (2017). Qualidade de vida na terceira idade em projeto de exercício físico em Ubá-MG. Revista Científica FAGOC-Saúde, 2(1), 21-27.

Arruda, M. F. D., Bazaglia, J. A., Saravalli, G., Cassettari, L. L., \& Souza, H. R. (2014). Ganho de força e função em idosos por treino isométrico com e sem resposta visual. Revista Brasileira de Medicina do Esporte, 20(4), 309-314. doi: http://dx.doi. org/10.1590/1517-86922014200401967

Benedetti, T. R. B., Mazo, G. Z., \& Borges, L. J. (2012). Condições de saúde e nível de atividade física em idosos participantes e não participantes de grupos de convivência de Florianópolis. Ciência \& Saúde Coletiva, 17(8), 2087-2093. doi: http://dx.doi.org/10.1590/ S1413-81232012000800019

Brucki, S. M., Nitrini, R., Caramelli, P., Bertolucci, P. H. F., \& Okamoto, I. H. (2003). Sugestões para o uso do Mini-Exame do Estado Mental no Brasil. Arquivos de Neuro-Psiquiatria, 61(3), 777-781. doi: http://dx.doi.org/10.1590/S0004-282X2003000500014

Dawalibi, N. W., Goulart, R. M. M., \& Prearo, L. C. (2014). Fatores relacionados à qualidade de vida de idosos em programas para a terceira idade. Ciência \& Saúde Coletiva, 19(8), 3505-3512. doi: http://dx.doi.org/10.1590/1413-81232014198.21242013 
Diener, E. D., Emmons, R. A., Larsen, R. J., \& Griffin, S. (1985). The satisfaction with life scale. Journal of Personality Assessment, 49(1), 71-75.

Folstein, M. F., Folstein, S. E., \& McHugh, P. R. (1975). "Mini-mental state": A practical method for grading the cognitive state of patients for the clinician. Journal of Psychiatric Research, 12(3), 189-198. doi: https://doi.org/10.1016/0022-3956(75)90026-6

Formiga, L. M. F., Oliveira, E. A. R., Borges, E. M., Santos, K. N. C., Araújo, A. K. S., \& Formiga, R. C. F. (2017). Envelhecimento ativo: Revisão integrativa. Revista Interdisciplinar Ciências e Saúde, 4(2), 9-18. Disponível em http://www.ojs.ufpi.br/index.php/rics/article/ view/3854

Freitas, V., Melo, C. C., Leopoldino, A., Boletini, T., \& Noce, F. (2018). Influencia de la actividad física sobre el estrés emocional en ancianos residentes en la comunidad. Revista de Psicologia del Deporte, 27(4), 75-81. Disponível em https://www.rpd-online.com/article/ view/v27-s1-freitas-carvalho-de-etal

Guimarães, A. C. A., Pedrini, A., Matte, D. L., Monte, F. G., \& Parcias, S. R. (2017). Ansiedade e parâmetros funcionais respiratórios de idosos praticantes de dança. Fisioterapia em Movimento, 24(4), 683-688. doi: http://dx.doi.org/10.1590/S0103-51502011000400012 Joia, L. C., Ruiz, T., \& Donalisio, M. R. (2007). Condições associadas ao grau de satisfação com a vida entre a população de idosos. Revista de Saúde Pública, 41, 131-138. doi: http:// dx.doi.org/10.1590/S0034-89102007000100018

Krug, R. D. R., Lopes, M. A., Balbé, G. P., Marchesan, M., \& Mazo, G. Z. (2016). Prevalence of commuting physical activity and associated factors in long-lived older adults. Revista Brasileira de Cineantropometria \& Desempenho Humano, 18(5), 520-529. doi: https:// doi.org/10.5007/1980-0037.2016v18n5p520

Matsudo, S., Araújo, T., Matsudo, V., Andrade, D., Andrade, E., Oliveira, L. C., \& Braggion, G. (2001). Questionário Internacional de Atividade Física (IPAQ): Estudo de validade e reprodutibilidade no Brasil. Revista Brasileira de Atividade Física \& Saúde, 6(2), 5-18. Disponível em http://rbafs.org.br/RBAFS/article/view/931

Melo, C. C. D., Costa, V. T. D., Boletini, T. L., Freitas, V. G. D., Costa, I. T. D., Arreguy, A. V., \& Noce, F. (2017). A influência do tempo de prática de dança de salão nos níveis de depressão de idosos. Revista de Psicología del Eeporte, 27(4), 67-73. Disponível em https://psycnet.apa. org/record/2018-28945-009

Mendes, G. A. B., Carvalho, M. V. D., Silva, A. M. T. C., \& Almeida, R. J. D. (2017). Relação entre atividade física e depressão em idosos: Uma revisão integrativa. Revista de Atenção à Saúde, 15(53), 110-116. doi: https://doi.org/10.13037/ras.vol15n53.4524

Neri, A.L. (2001). O fruto das sementes: Processos de amadurecimento e envelhecimento. In A. L. Neri (Org.), Desenvolvimento e envelhecimento: Perspectivas biológicas, psicológicas e sociais. Campinas, SP: Papirus.

Neto, F. (1999). Satisfação com a vida e características de personalidade. Psychologica, 22, 55-70. Disponível em https://www.uc.pt/fpce/psychologica/indexes/ind22

Oliveira, G. F., Barbosa, G. A., de Souza, L. E. C., da Costa, C. L. P., Araújo, R. D. C. R., \& Gouveia, V. V. (2009). Satisfação com a vida entre profissionais da saúde: Correlatos demográficos e laborais. Revista Bioética, 17(2), 319-334. Disponível em https://www.redalyc.org/ articulo.oa?id=361533249013 
Robins, L. M., Hill, K. D., Finch, C. F., Clemson, L., \& Haines, T. (2018). The association between physical activity and social isolation in community-dwelling older adults. Aging \& mental health, 22(2), 175-182. doi: https://doi.org/10.1080/13607863.2016.1242116

Rocha, C. A. Q. C., Guimarães, A. C., Borba-Pinheiro, C. J., Souza Santos, C. A., Moreira, M. H. R., Mello, D. B., \& Dantas, E. H. M. (2017). Efeitos de 20 semanas de treinamento combinado na capacidade funcional de idosas. Revista Brasileira de Ciências do Esporte, 39(4), 442-449. Disponível em https://www.sciencedirect.com/science/article/ pii/S0101328916300245?via\%3Dihub

Scarabottolo, C. C., Garcia Júnior, J. R., Gobbo, L. A., Alves, M. J., Ferreira, A. D., Zanuto, E. A. C., ... \& Christofaro, D. G. D. (2017). Influence of physical exercise on the functional capacity in institutionalized elderly. Revista Brasileira de Medicina do Esporte, 23(3), 200203. doi: http://dx.doi.org/10.1590/1517-869220172303150175

Sheppard, A. (1981). Attitudes towards aging: analysis of an attitudes inventory for younger adults: Abstracted. Catalog of Selected Documents in Psychology, 11, 9-27.

Silva, I. A., Amorim, J. R., Carvalho, F. T., \& Mesquita, L. S. A. (2017). Efeito de um protocolo de Facilitação NeuromuscularProprioceptiva(FNP) noequilíbrio postural deidosas. Fisioterapia e Pesquisa, 24(1), 62-67. doi: http://dx.doi.org/10.1590/1809-2950/16636724012017

Sposito, G., D’Elboux, M. J., Neri, A. L., \& Guariento, M. E. (2013). A satisfação com a vida e a funcionalidade em idosos atendidos em um ambulatório de geriatria. Ciência \& Saúde Coletiva, 18(12), 3475-3482. doi: http://dx.doi.org/10.1590/S1413-81232013001200004 Tarallo, R. D. S., Neri, A. L., \& Cachioni, M. (2017). Atitudes de idosos e de profissionais em relação a trocas intergeracionais. Revista Brasileira de Geriatria e Gerontologia, 20(3), 423-431. Disponível em http://www.scielo.br/pdf/rbgg/v20n3/pt_1809-9823rbgg-20-03-00421.pdf

Teixeira, C. M., Nunes, F. M. S., Ribeiro, F. M. S., Arbinaga, F., \& Vasconcelos-Raposo, J. (2016). Atividade física, autoestima e depressão em idosos. Cuadernos de Psicología del deporte, 16(3), 55-66. Disponível em http://scielo.isciii.es/scielo. php?pid=S1578-84232016000300006\&script=sci_abstract\&tlng=pt

Tomomitsu, M. R. S. V., Perracini, M. R., \& Neri, A. L. (2014). Fatores associados à satisfação com a vida em idosos cuidadores e não cuidadores. Ciência \& Saúde Coletiva, 19, 34293440. doi: http://dx.doi.org/10.1590/1413-81232014198.13952013

Recebido em: 18/06/2018 Revisado em: 08/02/2019 Aceite final: 27/02/2019

\section{Sobre os autores:}

Daniel Vicentini de Oliveira - Doutor em Gerontologia (UNICAMP). Mestre em Promoção da Saúde (UNICESUMAR). Especialista em Gerontologia pela Sociedade Brasileira de Geriatria e Gerontologia (SBGG). Graduado em Educação Física e em Fisioterapia (UNICESUMAR). E-mail: d.vicentini@hotmail.com, Orcid: http://orcid.org/0000-0002-0272-9773

Jonatã Felipe Queiroz de Souza - Graduado em Educação Física (UNIFAMMA). E-mail: jonatafelipe93@hotmail.com, Orcid: http://orcid.org/0000-0002-7254-1741 
Carla Thamires Laranjeira Granja - Graduanda em Educação Física (UNIVASF). E-mail: thamiresgranja1@gmail.com, Orcid: http://orcid.org/0000-0002-5859-0539

Mateus Dias Antunes - Mestre em Promoção da Saúde (UNICESUMAR). Especialista em Exercício Físico e Reabilitação de Idosos (UNIFAMMA). Graduado em Fisioterapia (UNICESUMAR). E-mail: mateus_antunes03@hotmail.com, Orcid: http://orcid.org/0000-0002-2325-2548

José Roberto Andrade do Nascimento Júnior - Doutor em Educação Física (UEM). Graduado em Educação Física (UEM). E-mail: jroberto.jrs01@gmail.com, Orcid: http://orcid.org/0000-0003-3836-6967 